\title{
COPD, diagrams and traditions: time to move on?
}

\section{Jørgen Vestbo}

The non-proportional Venn diagram explaining the components of chronic obstructive pulmonary disease (COPD) has been a graphical "COPD evergreen" along the Fletcher-Peto curve ever since it was proposed by Snider. ${ }^{1}$ The diagram has very much been intended as a way of visualising the concepts behind the processes contributing to COPD, but over the last few years there has been a growing interest in quantifying the various components of the diagram. ${ }^{23}$ These studies have used registered administrative diagnoses or self-reported diagnoses with the possibilities that errors in diagnostic labelling may have introduced significant bias. In this issue of Thorax Marsh et $\mathrm{al}^{4}$ present a proportional Venn diagram based on clinical data, pulmonary function measurements and CT findings from an ongoing population study in New Zealand (see page 761).

Marsh et al found that asthma was a significant cause of COPD in their general population sample; $55 \%$ of patients with COPD fulfilled their criteria for asthma whereas $30 \%$ had questionnaire-derived chronic bronchitis and $32 \%$ had emphysema defined by CT or reduced transfer factor. The overwhelming-and somewhat surprising-impact of asthma on COPD is not substantially different from that found in the previous studies; ${ }^{23}$ nevertheless, it challenges our traditional views and deserves attention. In addition, a significant proportion of patients with COPD were never smokers $(36 \%$ overall, ranging from $13 \%$ among COPD patients with emphysema to $38 \%$ among COPD patients with asthma).

The quick and easy interpretation of these findings is that asthma is the predominant cause of COPD and that we should stop viewing COPD as a disease closely related with smoking. For one thing, this would definitely change the view of COPD, as asthma-in contrast to complications of smoking-can hardly be said to be self-inflicted! However, as with most other simple

Correspondence to: Professor J Vestbo, Respiratory Research Group, ERC Building, 2nd Floor, Wythenshawe Hospital, Southmoor Road, Manchester M23 9LT, UK; jorgen.vestbo@manchester.ac.uk views, the concept of COPD as mainly persistent asthma with an irreversible component is likely to be misleading.

First, COPD is defined simply as a postbronchodilator forced expiratory volume in $1 \mathrm{~s} /$ forced vital capacity $\left(\mathrm{FEV}_{1} / \mathrm{FVC}\right.$ ) ratio $<0.7$ and no known diagnosis of bronchiectasis or tuberculosis. This is quoted as being taken directly from current guidelines such as the GOLD guidelines, ${ }^{5}$ but the guideline actually has a section dealing with COPD and asthma, patients with overlapping features and the differences in underlying airway inflammation. Although the guideline does not come up with clear guidance on how to label these patients, it does not recommend that every patient with asthma with a post-bronchodilator $\mathrm{FEV}_{1} / \mathrm{FVC}$ ratio $<0.7$ should be labelled as having COPD.

Second, the criteria for asthma are equally questionable. Marsh et al use a doctor's diagnosis of asthma or bronchodilator reversibility of $>15 \%$. Diagnostic labelling as a cause of bias may vary substantially from region to region and, at least in places other than New Zealand, patients with mild symptoms and airflow obstruction may be given an initial diagnosis of asthma even if the diagnosis after months of treatment with little effect on lung function turns out to be COPD. Also, the prevalence of asthma varies and is notoriously high in New Zealand. ${ }^{6}$ There is no doubt that asthma is characterised by variable airflow obstruction and therefore bronchodilator reversibility. There is at the same time no doubt that reversibility can be demonstrated in patients with COPD without any other features of asthma, and to most clinicians it seems intuitively wrong to state that these patients have asthma merely because of one measure of a feature of airflow obstruction that is known to be highly variable.

However, these points of criticism are unlikely to account for all of the findings in the paper by Marsh et al. It seems to have been a feature of COPD epidemiology that focus has to a high degree been on smoking as this is the major exposure relevant for COPD. This traditional approach has probably led to over-interpretation of the impact of smoking on COPD, as pointed out recently by the same authors. ${ }^{8}$ In a recent 25 -year follow-up study we found that $9 \%$ of never smokers, $11 \%$ of exsmokers and $31 \%$ of continuous smokersall with normal lung function at baselinedeveloped COPD over a 25 -year period. ${ }^{9}$ Nevertheless, whereas a clinician may be intrigued by the cause of COPD in the never smoker, he/she easily ascribes all COPD in smokers to the smoking habit despite the fact that a proportion of smokers would have had COPD even if they had not smoked. Data from the Burden of Lung Diseases programme have also clearly demonstrated a larger than traditionally expected prevalence of COPD among never smokers. ${ }^{10}$

In a larger perspective, there are two areas where the findings from the study by Marsh et al could therefore potentially have implications. The findings come from epidemiology, and this research discipline often impacts on disease prevention. Here, the message is quite clear. First, smoking cessation is essential for many reasons, including the prevention of COPD. Second-and clear from the paper by Marsh et $a l^{4}$ - prevention of irreversible airflow obstruction includes better diagnosis, treatment and follow-up of asthma. For the clinicians, however, the implications are probably less important. In the clinical setting, little time is usually spent on thinking about the components of COPD in general. Instead, the focus is on the individual patient, the patient's clinical characteristics, and the choice of treatment and secondary/tertiary prevention for the patient. As part of this process the clinician will make his/her own evaluation of the presence of previous asthma and features of current asthma and take these into account when making clinical decisions. Asthma with not fully reversible airflow obstruction will often continue to be treated like asthma and there are data to support this clinical practice. ${ }^{11}$ For research there is also a takehome message from this study. There is currently huge interest in the development of biomarkers in COPD, often with the aim of identifying patient groups with different natural histories. ${ }^{12}{ }^{13}$ Biomarkers are often thought of as substances measurable in blood, sputum or exhaled air, but the study by Marsh et al points out the value of clinicians' evaluation as biomarkers for defining phenotypes of COPD and thus underscores the role of the clinician in this type of translational research. 
Finally, is the non-proportional Venn diagram really a useful way of illustrating the components of COPD? Often the components of COPD are described as alveolar destruction (emphysema), small airway fibrosis (bronchiolitis) and chronic bronchitis. As most of the airflow obstruction is due to reduced airflow in the small conducting airways, ${ }^{14}$ it seems a little odd not to have small airways disease in the diagram. Should we then start working with an updated Venn diagram? Probably not. Perhaps the problem is that the Venn diagram should never have been taken too literally. It can stimulate thought and illustrate the heterogeneity of COPD; however, the effect of adding numbers to the diagram should motivate us to move on and try to gain new insights into this disease by coming up with novel concepts and potentially new subgroups of COPD which we had no idea ever existed.
Competing interests: None.

Thorax 2008;63:755-756. doi:10.1136/thx.2007.092924

\section{REFERENCES}

1. Snider G. Chronic obstructive pulmonary disease: a definition and implications of structural determinants of airflow obstruction for epidemiology. Am Rev Respir Dis 1989;140(3 Pt 2):S3-8.

2. Soriano JB, Davis KJ, Coleman B, et al. The proportional Venn diagram of obstructive lung disease: two approximations from the United States and the United Kingdom. Chest 2003;124:474-81.

3. Viegi G, Matteelli G, Angino A, et al. The proportional Venn diagram of obstructive lung disease in the Italian general population. Chest 2004;126:1093-101.

4. Marsh SE, Travers J, Weatherall M, et al. Proportional classifications of COPD phenotypes. Thorax 2008;63:761-7.

5. Global Initiative for Chronic Obstructive Lung Disease (GOLD). Global strategy for the diagnosis, management, and prevention of chronic obstructive pulmonary disease. Updated 2007. http://www. goldcopd.com (accessed 5 June 2008).

6. Masoli M, Fabian D, Holt S, et al. The Global Burden of Asthma Report (2004), Global Initiative for Asthma
(GINA). http://www.ginasthma.com (accessed 5 June 2008)

7. Calverley PMA, Burge PS, Spencer S, et al. Bronchodilator reversibility testing in chronic obstructive pulmonary disease. Thorax 2003;58:659-64.

8. Marsh S, Aldington S, Shirtcliffe P, et al. Smoking and COPD: what really are the risks? Eur Respir J 2006;28:883-4.

9. Lokke A, Lange $P$, Scharling $H$, et al. Developing COPD: a 25 years follow-up study of the general population. Thorax 2006;61:935-9.

10. Buist AS, McBurnie MA, Vollmer WM, et al. International variation in the prevalence of COPD (the BOLD study): a population-based prevalence study. Lancet 2007;370:741-50.

11. Fabbri LM, Romagnoli M, Corbetta L, et al. Differences in airway inflammation in patients with fixed airflow obstruction due to asthma or chronic obstructive pulmonary disease. Am J Respir Crit Care Med 2003;167:418-24.

12. Stockley RA. Biomarkers in COPD: time for a deep breath. Thorax 2007;62:657-60.

13. Vestbo J, Anderson W, Coxson HO, et al. Evaluation of COPD Longitudinally to Identify Predictive Surrogate Endpoints (ECLIPSE). Eur Respir J 2008;31:869-73.

14. Hogg JC, Macklem PT, Thurlbeck WM. Site and nature of airway obstruction in chronic obstructive lung disease. N Engl J Med 1968;278:1355-60.

\section{What evidence could validate the definition of COPD?}

\section{Roberto de Marco}

Chronic obstructive pulmonary disease (COPD) is one of the leading causes of disability and death in both the developed and developing worlds, ${ }^{1-3}$ and it is largely underdiagnosed. ${ }^{4}$

While it is generally accepted that the ratio between forced expiratory volume in $1 \mathrm{~s}\left(\mathrm{FEV}_{1}\right)$ and forced vital capacity (FVC) or slow vital capacity (SVC) is the most important measure that characterises airflow obstruction, ${ }^{5}{ }^{6}$ there is still no consensus on what the best definition of COPD should be.

In an attempt to simplify the diagnosis of COPD, improve the detection of the disease in primary care and standardise methods to measure the prevalence of COPD in different countries, the Global

Correspondence to: Professor R de Marco, Unit of Epidemiology and Medical Statistics, Department of Medicine and Public Health, Strada le Grazie 8, 37134 Verona, Italy; roberto.demarco@univr.it
Initiative for Chronic Obstructive Lung Disease (GOLD) has defined COPD as a post-bronchodilator $\mathrm{FEV}_{1} / \mathrm{FVC}$ ratio $<0.70 .^{5}$

As the $\mathrm{FEV}_{1} / \mathrm{FVC}$ ratio decreases with age, this fixed ratio has been criticised as it may overdiagnose the disease in elderly subjects ${ }^{7}$ and underdiagnose it in young adults. ${ }^{8}$ For this reason, the ATS/ERS guidelines on lung function testing ${ }^{9}$ propose using the lower limit of normality (LLN) instead of a fixed cut-off to define COPD. LLN is the lower fifth percentile of the frequency distribution of the $\mathrm{FEV}_{1} /$ FVC ratio in a healthy population of a given sex and age. The guidelines also point out that the SVC could be more accurate than FVC to diagnose airflow obstruction

A hot debate between the supporters of the two different points of view is currently ongoing, as can be seen in scientific journals. ${ }^{10} 11$ As an epidemiologist I believe there could be sound reasons to support both approaches but, in my opinion, there is insufficient evidence to make one preferable to the other. The positive aspect of the GOLD definition is simplicity and, thanks to this, its use is quite widespread. It relies entirely on the opinions of clinical experts and assumes that an $\mathrm{FEV}_{1} / \mathrm{FVC}$ ratio $<0.70$ is, "on average", a good threshold to discriminate patients with clinically defined COPD from normal subjects. The LLN definition is in theory more rational; it is based on the statistical approach used to derive the reference (or "normal") ranges for biological variables. It depends entirely on the accuracy of "reference equations" (to my knowledge, accurate national reference equations do not currently exist in many cases) and it assumes that (1) everyone outside the range is affected by COPD and (2) all patients affected by COPD are outside the range (in other words, the distribution of diseased and healthy people does not overlap).

The trait that the two approaches have in common is that the definition of the disease is based exclusively on a variablethe $\mathrm{FEV}_{1} / \mathrm{FVC}$ (or $\mathrm{FEV}_{1} / \mathrm{SVC}$ ) - that is distributed along a continuum in the general population. Any definition of COPD based on a dichotomy of this 